\title{
Pulmonary valve papillary fibroelastoma in an asymptomatic young patient
}

\begin{abstract}
Among primary cardiac tumors (prevalence $0.0017-0.28 \%$ ), cardiac papillary fibroelastoma (CPF) is uncommon, $8 \%$ of cases, but it's the most frequent cardiac neoplasm originating from the endocardium. It usually involves left-sided heart valves, very rarely the pulmonary valve. Echocardiographically this neoplasm is a small mass, $<20 \mathrm{~mm}$, almost $50 \%$ with a small stalk, and mobile. Macroscopically it appears similar to a "sea anemone". Clinically, $\mathrm{CPF}$ could be associated with coronary, cerebral, pulmonary and retinal emboli. We present the case of a 54year old man who came to our attention for the incidental discover at echocardiogram of a mobile mass attached to the ventricular side of the pulmonary valve. The patient underwent prompt surgical removal of the neoplasm through median sternotomy with sparing of the valve.
\end{abstract}

Keywords: papillary fibroelastoma, pulmonary valve, primary cardiac tumor
Volume I Issue $6-2014$

\author{
Luca Caprili, Enrico Amoncelli, Alberto \\ Albertini \\ Department of Cardiovascular and Thoracic Surgery, Salus \\ Hospital, Italy
}

Correspondence: Luca Caprili, Department of Cardiovascular and Thoracic Surgery, Salus Hospital, GVM-Care and Research, Via U. Levi 7, Reggio Emilia, Italy, Tel 39-0522-499I23, Email lukap70@libero.it

Received: November 25, 2014 | Published: November 27, 2014

\section{Introduction}

Cardiac papillary fibroelastoma (CPF) is an extremely rare primary cardiac neoplasm, only $8 \%$ of all primary cardiac tumors, but the most common neoplasm originating from the endocardium. The incidence of this pathology is estimated $0,019 \%$ in clinical series. ${ }^{1}$ Most frequently CPF involves left-sided heart valves $(89 \%)$, especially aortic valve (29\%), whereas mitral valve is less interested (25\%). Pulmonary valve is affected only for the $8 \%$ of all cases. Very seldom this pathology can present multiple tumors. The main age of presentation is between 50 and 60years. Echocardiographically it appears as a small mass, with dimensions between 10 and $20 \mathrm{~mm}$, in $50 \%$ of cases presenting a thin stalk attached to the valve, in the majority of cases mobile. ${ }^{2}$ (Figure 1). Macroscopically the aspect is characteristic, similar to a "sea anemone", with multiple frond-like extrusions (Figures $2 \& 3$ ). The histological examination shows dense elastin coated by collagen, lined by flat endocardial cells. The collagen is filled by smooth muscle cells, elastin, fibroblast and occasionally cysts and areas of hemorrhage. The cause of this pathology is still unclear, different hypothesis have been made: stress of blood flow across the valve producing endothelial damage and subsequent thrombosis with deposition of fibrin fibers; viral induction by cytomegalovirus; extension of Lambl's excrescences, iatrogenic (post radiation, surgery). ${ }^{3}$ The presence of CPF carries an actual risk of embolization, although not easily numerically definable. Its typical structure and mobility however strongly suggest prompt prevention of embolic complications and surgical exeresis.

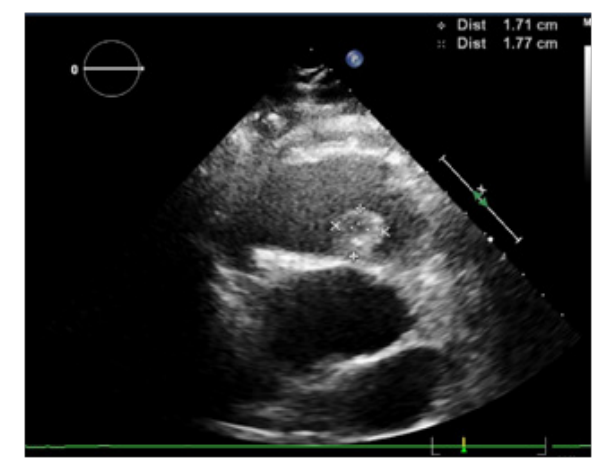

Figure I

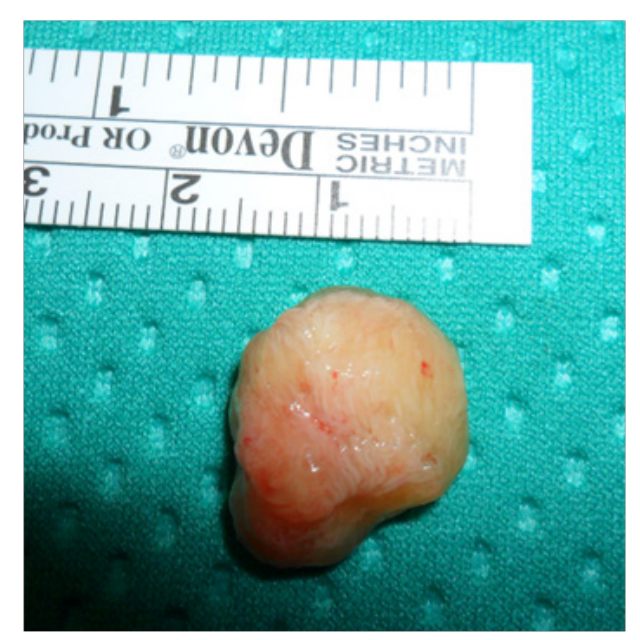

Figure 2

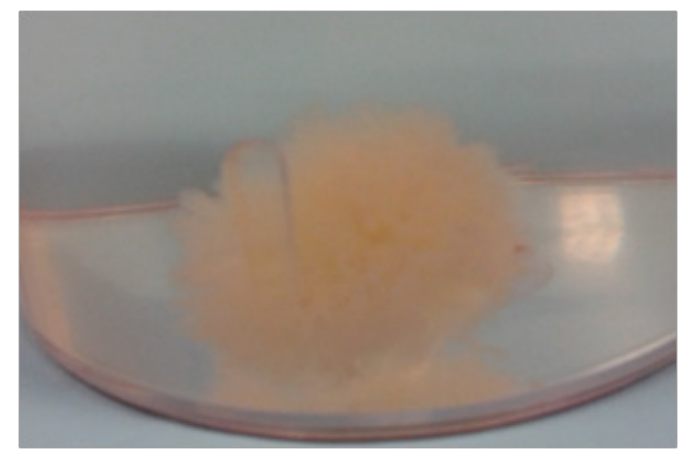

Figure 3

\section{Case report}

We describe the case of a 54year old male, completely asymptomatic, with hypertension as unique cardiac risk factor. During routine check-up a cardiac echo showed a grossly spherical mass attached to the ventricular side of the pulmonary valve; and was scheduled for trans esophageal echo at our Institution. The presence of 
the mass was confirmed, mobile across the valve during cardiac cycle, apparently arising from the ventricular surface of the posterior leaflet of the pulmonary valve. The dimensions were measured $17 \times 17 \mathrm{~mm}$. The ventricular function was normal, whereas the aortic valve showed up to be bicuspid, normally functioning, with a dilated ascending aorta $(45 \mathrm{~mm})$. In consideration of the extreme mobility of the mass and of its dimensions, carrying the risk of actual pulmonary embolization, the patient was promptly anticoagulated and after a few days operated. Through median sternotomy, on cardiopulmonary bypass with short aortic cross clamping (10minutes), the excision of the mass was performed through transversal incision of the pulmonary artery, with complete shaving and sparing of the valve. The postoperative course was uneventful and the patient was discharged on the $6^{\text {th }}$ postoperative day. The histopathological exam confirmed the diagnosis of CPF.

\section{Discussion}

$\mathrm{CPF}$ is a very rare entity; in this case it was discovered incidentally during routine examination of a young, completely asymptomatic individual, carrying a real risk of life threatening clinical manifestation as first symptom. The incidence of pulmonary embolism is not easy to determine precisely, due to the extreme rarity of this tumor. However embolization and thromboembolic complications, myocardial ischemia, infarction and stroke, pulmonary embolism and even ventricular fibrillation and sudden death have been reported. ${ }^{4}$ as symptoms that carried to diagnosis. The emboli may be determined by platelet clotting or thrombi organized on the surface of the mass, or be fragments of the mass itself. In one series of patient, ${ }^{2} 22 \%$ of patients who were treated without surgery experienced neurologic events, and $4 \%$ had peripheral embolic events during the follow-up period. Furthermore some studies report a 50\% CPF-related mortality in non-treated patients, due to systemic or pulmonary embolization or flow obstruction. ${ }^{5}$ Considering this scenario, in our opinion, once the diagnosis is made, prompt treatment is needed. Anticoagulation should start immediately to avoid embolic risk in all patients. Despite the scarcity of cases, accordingly to other authors, we consider the tumor mobility the most important factor that can determine high risk of embolization. ${ }^{6}$ subsequently, dimensions superior to $10 \mathrm{~mm}$, the location of the mass, the presence of other cardiac disease should guide the indication for surgery. Surgical procedure should be focused to the complete removal of the mass, avoiding its fragmentation, preferably sparing the valve. Intraoperative Trans esophageal echo is essential to confirm the complete exeresis of the mass and the valvular function.

\section{Conclusion}

In our opinion this rare cause of embolic stroke and other fatal complications, by means of appropriate use of imaging modality, preoperative anticoagulation and urgent surgical resection, can be successfully treated. The need for urgent surgery should be decided taking into accounts the characteristics of the mass and of the patient himself: mobility as the most important factor, and subsequently dimensions, location, presence of other cardiac diseases, age of the patient and risk of surgery.

\section{Acknowledgments}

None.

\section{Conflicts of interest}

Authors declare that there are no conflicts of interest.

\section{References}

1. Howard RA, Aldea GS, Shapira OM, et al. Papillary fibroelastoma: increasing recognition of a surgical disease. Ann Thorac Surg. 1999;68(5):1881-1885.

2. Klarich KW, Enriquez Sarano M, Gura GM, et al. Papillary fibroelastoma: echocardiographic characteristics for diagnosis and pathologic correlation. J AM CollCardiol.1997;30(3):784-790.

3. Jha NK, Khouri M, Murphy DM, et al. Papillary fibroelastoma of the aortic valve a case report and literature review. J Cardiothorac Surg. 2010;5:84.

4. Gowda RM, Khan IA, Nair CK, et al. Cardiacpapillary fibroelastoma:a comprehensive analysis of 725 cases. Am Heart J. 2003;146(3):404-410.

5. Sun JP, Asher CR, Yang XS, et al. Clinical and echocardiographic characteristics of papillary fibroelastomas: a retrospective and prospective study in 162 patients. Circulation. 2001;103(22):2687-2693.

6. Bouhzam N, Kurtz B, Doguet F, et al. Incidental Papillary Fibroelastoma Multimodal. Imaging and Surgical Decisions in 2 Patients. Tex Heart Inst J. 2012;39(5):731-735. 